\title{
Management von paraneoplastischem Pruritus
}

\author{
Brandon Rowe Gil Yosipovitch
}

Department of Dermatology and Itch Center, Temple University School of Medicine, Philadelphia, Pa., USA

\section{Zusammenfassung}

Paraneoplastischer Pruritus tritt als systemische Reaktion auf eine zugrunde liegende maligne Erkrankung auf. Paraneoplastischer Pruritus ist am häufigsten mit malignen lymphoproliferativen Erkrankungen sowie soliden Tumoren, die zu Cholestase führen, assoziiert. Paraneoplastischer Pruritus kann sich sowohl ohne primären Hautausschlag manifestieren als auch in Verbindung mit dermatologischen Erkrankungen wie Erythrodermie, Acanthosis nigricans, Dermatomyositis (DM), transitorischer akantholytischer Dermatose (Morbus Grover) oder eruptiver seborrhoischer Kerato- se. Die Behandlung des paraneoplastischen Pruritus beruht primär auf der Behandlung der zugrunde liegenden malignen Erkrankung, die ursächlich für die systemische Reaktion ist. Wenn die maligne Erkrankung nicht auf Behandlung anspricht, können andere Therapien beim paraneoplastischen Pruritus wirksam sein, wie selektive Serotonin-Wiederaufnahmehemmer, Mirtazapin, Gabapentin, Thalidomid, Opioide, Aprepitant und Histon-Deacetylase-Inhibitoren.

c) 2016 S. Karger AG, Basel

\section{Definition und klinische Merkmale}

Paraneoplastischer Pruritus ist definiert als Juckreiz, der entweder im Verlauf einer malignen Erkrankung auftritt oder der Diagnosestellung vorangeht [1]. Wenn eine direkte neoplastische Invasion oder Kompression durch einen Tumor die Ursache für Juckreiz ist, gilt dies nicht als paraneoplastischer Pruritus. 2015 definierte die Special Interest Group des «International Forum on the Study of Itch» den paraneoplastischen Pruritus wie folgt: 〈Juckreiz als systemische (nicht lokale) Reaktion auf das Vorliegen eines Tumors oder einer malignen hämatologischen Erkrankung, der weder durch lokal vorhandene Tumorzellen noch durch die Tumortherapie hervorgerufen wird> [2].

Zur Prävalenz des paraneoplastischen Pruritus liegen nur begrenzte epidemiologische Daten vor. Eine retrospektive Studie, in der 700 Patienten mit soliden Tumoren und malignen hämatologischen Erkrankungen untersucht wurden, ergab für generalisierten Juckreiz bei Patienten mit begleitenden dermatologischen Manifestationen eine Prävalenz von 13\% [3]. Die größten prospektiven Studien wurden an Patienten durchgeführt, die an chronischem Juckreiz ohne sichtbare Hautmanifestationen litten. In einer Studie wurden 8743 Patienten mit chronischem
Juckreiz ohne Ausschlag untersucht. Die Analyse ergab bei diesen Patienten im Vergleich zur Allgemeinbevölkerung eine Verdoppelung des Risikos, eine maligne hämatologische Erkrankung zu entwickeln, und ein 3-fach erhöhtes Risiko für ein Malignom der Gallengänge [4]. Eine ähnliche Studie, in der 12813 Patienten mit chronischem Juckreiz begleitet wurden, ermittelte im Vergleich zur Allgemeinbevölkerung einen Anstieg der Inzidenz von Krebserkrankungen um insgesamt $13 \%$ und von hämatologischen Krebserkrankungen um 68\% über einen Zeitraum von 5 Jahren [5]. Paraneoplastischer Pruritus kommt am häufigsten bei lymphoproliferativen malignen Erkrankungen vor, unter anderem bei multiplem Myelom, Non-Hodgkin-Lymphomen (15\% Prävalenz) und Hodgkin-Lymphomen (25\% Prävalenz) [6-8].

Mit paraneoplastischem Pruritus assoziierte hämatologische Malignome und solide Tumoren, die zu Cholestase führen, gehen oft nicht mit Hautveränderungen einher [1, 7]. Es können jedoch verschiedene beobachtbare mit Juckreiz verbundene Hautveränderungen infolge einer malignen Erkrankung auftreten (Abb. 1), darunter Erythrodermie [9], Acanthosis nigricans, Dermatomyositis (DM), transitorische akantholytische Dermatose (Morbus Grover) sowie eruptive seborrhoische Keratosen.

\section{KARGER}

Fax +497614520714

information@karger.com

www.karger.com

\section{(c) 2018 S. Karger GmbH, Freiburg}

Accessible online at:

www.karger.com/kkd
Gil Yosipovitch, MD

Department of Dermatology and Itch Center, Temple University School of Medicine

3322 North Broad Street, Medical Office Building, Suite 212

Philadelphia, PA 19140 (USA)

gil.yosipovitch@tuhs.temple.edu 
Abb. 1. a, b Ein 71-jähriger männlicher Patient entwickelte eine erworbene Palmoplantarkeratose, danach manifestierten sich an den Beinen hyperpigmentierte Flecken, die als Mycosis fungoides diagnostiziert wurden. Der Patient klagte über intensiven generalisierten Juckreiz, der einem paraneoplastischen Pruritus infolge der Mycosis fungoides entsprach und sich unter Ganzkörper-Elektronenbestrahlung der Haut besserte.
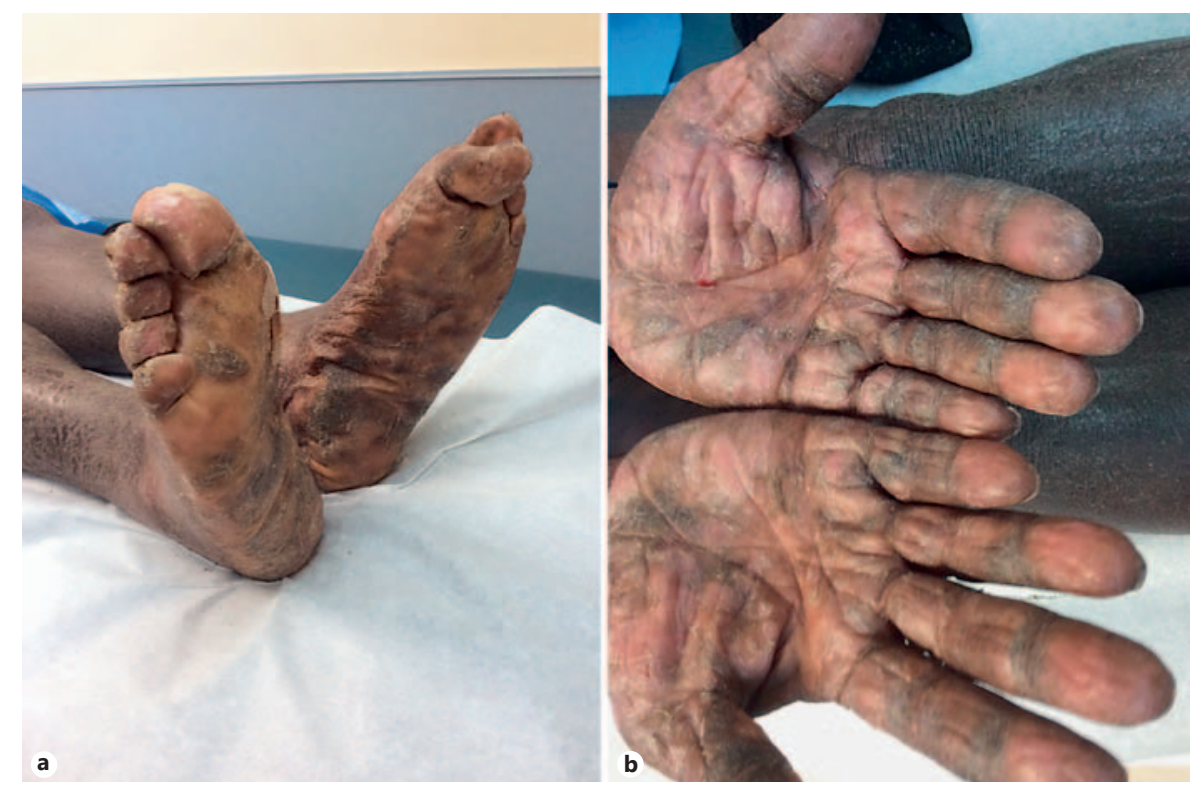

Acanthosis nigricans ist eine häufig vorkommende Hauterkrankung, die durch samtige, hyperpigmentierte Plaques vor allem am Hals und in den Achselhöhlen gekennzeichnet ist. Eine retrospektive Analyse von 90 Patienten mit Acanthosis nigricans ergab, dass 17 von ihnen (19\%) an einer assoziierten malignen Erkrankung litten und sieben an generalisiertem Juckreiz, während bei weiteren drei Patienten dem generalisierten Juckreiz die Manifestation einer Acanthosis nigricans vorausging [10].

DM ist eine Autoimmunerkrankung der Haut, die durch proximale Muskelschwäche, heliotropen Ausschlag, Photosensitivität und Gottron-Papeln gekennzeichnet ist. Sie ist mit verschiedenen malignen Erkrankungen in Zusammenhang gebracht worden, darunter Kolon-, Ovarial- und Mammakarzinome. Eine groß angelegte populationsbasierte Studie aus Schweden ergab, dass von 392 untersuchten Patienten mit DM 59 (15\%) eine assoziierte Krebserkrankung hatten, die gleichzeitig mit der DM oder später diagnostiziert wurde [11]. Im Vergleich zur Allgemeinbevölkerung wurde ein relatives Risiko für eine Krebserkrankung von 2,4 (95-\%-Konfidenzintervall (KI): 1,6-3,6) bei Männern und 3,4 (95\%-KI: 2,4-4,7) bei Frauen ermittelt. In einer prospektiven Studie zum Vergleich der klinischen Merkmale der DM in den USA und in Singapur entwickelten in einem Zeitraum von fünf Jahren drei der DM-Patienten aus Singapur eine maligne Erkrankung, in zwei dieser Fälle ein nasopharyngeales Karzinom [12].

Eine Querschnittsstudie zur Prävalenz von Pruritus bei DM-Patienten ergab, dass $85 \%$ der Studienteilnehmer an Juckreiz von unterschiedlichem Schweregrad litten und in 58\% der Fälle von mittlerem bis hohem Schweregrad [13]. Weitere Studien haben bestätigt, dass Juckreiz ein verbreitetes Symptom bei DM ist [12, 14]. Transitorische akantholytische Dermatose ist eine weitere Kategorie juckender Hautläsionen, die mit malignen Erkrankungen assoziiert ist. Sie ist durch einen mit Pruritus verbundenen papulovesikulären Ausschlag am Oberkörper gekennzeichnet [15]. In der Regel klingt sie nach einigen Wochen bis Monaten spontan wieder ab. Berichten zufolge kann transitorische akantholytische
Dermatose bei oder nach der Diagnose von chronischer und akuter myeloischer Leukämie, Karzinomen des Übergangsepithels der Harnblase und Nieren sowie multiplem Myelom auftreten [16].

Das Leser-Trélat-Zeichen wird als plötzlich in Größe und Anzahl zunehmende seborrhoische Keratosen im Rahmen einer inneren malignen Erkrankung beschrieben [17]. Generalisierter Juckreiz geht Berichten zufolge mit dem Auftreten des Leser-Trélat-Zeichens einher [18]. Ob das Leser-Trélat-Zeichen ein legitimes paraneoplastisches Krankheitszeichen ist, wird kontrovers diskutiert, unter anderem weil es typischerweise mit Acanthosis nigricans assoziiert ist [19]. Wie weiter oben dargelegt ist Acanthosis nigricans selbst ein paraneoplastisches Krankheitszeichen, was zu Confounding-Effekten führen kann, wenn es darum geht, die wahre Prävalenz maligner Erkrankungen bei Patienten mit LeserTrélat-Zeichen zu ermitteln. Darüber hinaus wurde bemerkt, dass das Leser-Trélat-Zeichen prädominant bei Patienten höheren Alters auftritt, also in der Altersgruppe, die am häufigsten maligne Erkrankungen entwickelt. Weiterführende statistische Analysen werden benötigt, um die wahre Epidemiologie von malignen Erkrankungen festzustellen, die mit dem Leser-Trélat-Zeichen assoziiert sind.

\section{Management}

Die wirksamste Behandlung von paraneoplastischem Pruritus ist die Therapie der zugrunde liegenden malignen Erkrankung. Für den Fall therapierefraktärer oder sich sehr langsam zurückbildender maligner Erkrankungen gibt es eine Reihe von Therapien zur Linderung des Juckreizes, die sich als wirksam erwiesen haben. Nachstehend wird das Spektrum der Behandlungsmöglichkeiten unter Berücksichtigung der Wirksamkeit, des Nebenwirkungsprofils und der Anwendungsgeschichte vorgestellt. Außer auf Wirkungen und Nebenwirkungen wird auch auf den vermuteten Wirkmechanismus eingegangen. 


\section{Selektive Serotonin-Wiederaufnahmehemmer}

Selektive Serotonin-Wiederaufnahmehemmer inhibieren gezielt die Wiederaufnahme von Serotonin im Nervensystem und verstärken so die Wirkung von ausgeschüttetem Serotonin. Der Mechanismus, über den diese Wirkstoffe Pruritus lindern, ist nicht abschließend geklärt. Eine Theorie lautet jedoch, dass sie diese Wirkung durch Modulation der zentralen Opioidrezeptoren erzielen [20]. In einer randomisierten klinischen Studie wurde festgestellt, dass paraneoplastischer Pruritus um 50\% abnahm, wenn den Patienten 7 Tage lang $20 \mathrm{mg}$ Paroxetin täglich verabreicht wurden [20]. Selektive Serotonin-Wiederaufnahmehemmer sind gut verträglich. Die hauptsächlichen Nebenwirkungen, die bei der genannten Dosis zu beobachten sind, sind Übelkeit und Benommenheit.

\section{Mirtazapin}

Mirtazapin ist ein Antagonist mehrerer Rezeptoren, dazu zählen die zentralen a2-adrenergen präsynaptischen Autorezeptoren sowie 5-HT2-, 5-HT3- und H1-Rezeptoren, die die Zielstrukturen von Antihistaminika sind. Es ist unklar, welcher dieser Rezeptoren für die antipruriginöse Wirkung von Mirtazapin verantwortlich ist. Jedoch zeigt der Wirkstoff nachweislich hohe Wirksamkeit in der Linderung oder vollständigen Elimination von paraneoplastischem Pruritus in Dosen von 7,5-30 mg jeden Abend [21]. Mirtazapin hat sich als besonders wirkungsvoll zur Behandlung von nächtlichem Juckreiz im Rahmen von chronischem Pruritus erwiesen, was ein wichtiger Aspekt für die Behandlungsplanung bei paraneoplastischem Pruritus sein kann [22]. Die wichtigsten Nebenwirkungen von Mirtazapin sind Sedierung und Gewichtszunahme [23, 24].

\section{Gabapentin}

Gabapentin ist ein strukturelles Analogon der $\gamma$-Aminobuttersäure (GABA). Es wird traditionell in der Behandlung neuropathischer Schmerzen eingesetzt, wo es durch Inhibition neuronaler Calciumkanäle zur Senkung der intrazellulären Glutamatkonzentration und zu verringerter neuronaler Erregbarkeit führt [25]. Die Hemmung des Juckreizes könnte über einen vergleichbaren Mechanismus erzielt werden, dies ist jedoch noch in spezifischen Untersuchungen zu klären. Gabapentin wurde Berichten zufolge erfolgreich zur Behandlung von Juckreiz eingesetzt, der durch kutanes T-Zell-Lymphom (CTCL) induziert war. Hierzu wird eine Anfangsdosis von $300 \mathrm{mg}$ jeden Abend und eine Höchstdosis von $2400 \mathrm{mg}$ pro Tag vorgeschlagen [26]. Gabapentin wird im Allgemeinen gut vertragen. Die am häufigsten gemeldeten Nebenwirkungen sind Schläfrigkeit (20\%), Ataxie (17\%), Nystagmus (15\%) und Kraftlosigkeit [27, 28].

\section{Thalidomid}

Thalidomid wurde früher zur Behandlung von Morgenübelkeit in der Schwangerschaft eingesetzt und gelangte zu unrühmlicher
Bekanntheit, weil es Fehlbildungen der Extremitäten hervorrief. Seitdem hat es sich als Chemotherapeutikum etabliert und ist auch in der Bekämpfung von Juckreiz nachweislich wirksam. Sein Wirkmechanismus ist unbekannt. Es inhibiert jedoch bekannterweise Tumornekrosefaktor- $a$, Interleukin(IL)6, IL-10 und IL-12, und es hat eine zentral dämpfende Wirkung, die für die beobachteten antipruriginösen Eigenschaften verantwortlich sein könnte [29]. Thalidomid zeigt Wirkung in der Behandlung von Pruritus bei Hodgkin-Lymphom [30] und es ist eine ausgezeichnete Behandlungsoption für Patienten, die an Juckreiz infolge von multiplem Myelom leiden, da Thalidomid auch zur Behandlung von multiplen Myelomen, die refraktär gegenüber Standard-Chemotherapeutika waren, erfolgreich eingesetzt worden ist [31]. Aufgrund der umfassend dokumentierten teratogenen Wirkung auf die Entwicklung der fetalen Gliedmaßen ist Thalidomid jedoch Frauen, die Kinder bekommen können, nur nach besonders sorgfältiger Abwägung zu verordnen. Ein Screening auf periphere Neuropathie sollte bei jedem Patienten durchgeführt werden, der Thalidomid über mehr als 3 Monate anwendet, da eine 2-Jahres-Inzidenz peripherer Neuropathien von $25-56 \%$ festgestellt wurde [32].

\section{Opioide}

к-Opioidagonisten haben sich als wirksame Antipruriginosa erwiesen [33, 34]. Das Analgetikum Butorphanol, ein gemischter $\mu$-Opioidantagonist/ $\kappa$-Opioidagonist, vermag Berichten zufolge Juckreiz infolge eines Non-Hodgkin-Lymphoms rasch zu lindern [35]. Ein weiteres Opioid mit ähnlichen Eigenschaften ist Nalbuphin, ein gemischter $\mu$-/ $\kappa$-Opioidagonist, der sowohl als Analgetikum als auch als Antipuriginosum Wirkung gezeigt hat. Eine aktuelle Metaanalyse randomisierter kontrollierter klinischer Studien ergab keinen signifikanten Unterschied in der Schmerzlinderung zwischen Nalbuphin und Morphin; jedoch litten die mit Morphin behandelten Patienten mit 5-mal höherer Wahrscheinlichkeit an Juckreiz als die Patienten, die Nalbuphin erhielten [36]. Aktuelle klinische Studien belegen die Wirksamkeit von Nalbuphin in der Linderung von Morphin-induziertem Juckreiz und urämischem Pruritus $[37,38]$. Wie auch bei anderen Opioiden zählen zu den häufigen Nebenwirkungen Übelkeit, Sedierung und Verwirrtheit.

\section{Aprepitant}

Aprepitant ist ein Neurokinin-1-Rezeptorantagonist, der ursprünglich als Antiemetikum für Chemotherapiepatienten angewendet wurde. In Mausmodellen konnte gezeigt werden, dass Neurokinin-1-Rezeptorantagonisten Juckreiz blockieren, der durch Substanz P hervorgerufen wird. Substanz P ist ein sensorisches Neuropeptid, das nachweislich mit dem Schweregrad von Ekzem-Erkrankungen korreliert [39, 40]. Aprepitant in einer Dosierung von $80 \mathrm{mg}$ täglich führte zur wirksamen Linderung von Juckreiz bei Patienten mit Sézary-Syndrom, die mit schwerer pruritusbedingter Schlaflosigkeit und Depression stationär behandelt wurden [42]. Die Patienten sprachen schnell auf die Be- 
handlung an. Die durchschnittliche Schwere des Juckreizes ging nach nur einem Tag von 8 auf 2,3 auf einer 10-stufigen visuellen Analogskala für Pruritus zurück. Die häufigsten Nebenwirkungen im Zusammenhang mit Aprepitant sind Übelkeit, Diarrhö und Obstipation.

\section{Histon-Deacetylase-Inhibitoren}

Histon-Deacetylase-Inhibitoren wie z.B. Vorinostat und Romidepsin werden seit neuestem zur Behandlung von CTCL untersucht. Die Bildung von Histon ist eine Voraussetzung für die Herstellung von Chromatin - ein kritischer Vorgang maligner T-Zellen mit hoher Teilungsrate. Durch die Reduktion maligner T-Zellen sinkt auch die Ausschüttung von Chemokinen, die mit der Pathophysiologie des paraneoplastischen Pruritus bei CTCLPatienten in Zusammenhang gebracht werden. Besonders IL-31, ein von CD4-Helfer-T-Zellen hergestelltes Chemokin, korreliert Untersuchungen zufolge mit Pruritus bei CTCL [42]. Eine Reduktion der IL-31-Serumkonzentration bei Patienten mit CTCL geht nachweislich signifikant mit vermindertem Juckreiz einher [43].
Histon-Deacetylase-Inhibitoren können bei CTCL-Patienten in Betracht gezogen werden, die an starkem Juckreiz leiden, um den Pruritus und zugleich die zugrunde liegende maligne Erkrankung zu behandeln. Die häufigsten Nebenwirkungen sind Übelkeit (81\%) und Erschöpfung (69\%). Aufgrund von Berichten über erhöhte Leberenzymwerte, Neutropenie und virale Reaktivierung ist bei Anwendung von Histon-Deacetylase-Inhibitoren außerdem die routinemäßige klinische und laboranalytische Überwachung der Patienten erforderlich [44].

\section{Schlussfolgerung}

Zahlreiche Therapien haben Wirksamkeit in der Behandlung von paraneoplastischem Pruritus gezeigt. Wir haben hier das Spektrum der Therapieoptionen bei paraneoplastischem Pruritus unter Berücksichtigung von Wirksamkeit, Nebenwirkungsprofil und Neuartigkeit des Wirkstoffs vorgestellt. Eine weitergehende Erforschung der Pathophysiologie des paraneoplastischen Pruritus wird auch zu zielgerichteteren Behandlungsmaßnahmen für die Patienten führen.

\section{Literatur}

1 Yosipovitch G: Chronic pruritus: a paraneoplastic sign. Dermatol Ther 2010;23:590-596.

$\checkmark 2$ Weisshaar E, Weiss M, Mettang T, et al: Paraneoplastic itch: an expert position statement from the Special Interest Group (SIG) of the International Forum on the Study of Itch (IFSI). Acta Derm Venereol 2015;95:261-265.

3 Kilic A, Gül U, Soylu S: Skin findings in internal malignant diseases. Int J Dermatol 2007;46: 1055-1060.

$\checkmark 4$ Fett N, Haynes K, Propert KJ, Margolis DJ: Five-year malignancy incidence in patients with chronic pruritus: a population-based cohort study aimed at limiting unnecessary screening practices. J Am Acad Dermatol 2014;70:651-658.

5 Johannesdottir SA, Farkas DK, Vinding GR, et al: Cancer incidence among patients with a hospital diagnosis of pruritus: a nationwide Danish cohort study. Br J Dermatol 2014;171: 839-846.

6 Erskine JG, Rowan RM, Alexander JO, Sekoni GA: Pruritus as a presentation of myelomatosis. Br Med J 1977;1:687-688.

7 Gobbi PG, Attardo-Parrinello G, Lattanzio G, et al: Severe pruritus should be a B-symptom in Hodgkin's disease. Cancer 1983;51:1934-1936.

8 Mandal S, Varma K, Jain S: Cutaneous manifestations in non-Hodgkin's lymphoma. Acta Cytol 2007;51:853-859.

9 Robak E, Robak T: Skin lesions in chronic lymphocytic leukemia. Leuk Lymphoma 2007;48: 855-865.

10 Brown J, Winkelmann RK: Acanthosis nigricans: a study of 90 cases. Medicine (Baltimore) 1968;47:33-51.

11 Sigurgeirsson B, Lindelöf B, Edhag O, Allander E: Risk of cancer in patients with dermatomyositis or polymyositis. A population-based study. N Engl J Med 1992;326:363-367.
12 Yosipovitch G, Tan A, LoSicco K, et al: A comparative study of clinical characteristics, workup, treatment, and association to malignancy in dermatomyositis between two tertiary skin centers in the USA and Singapore. Int J Dermatol 2013;52:813-819.

13 Shirani Z, Kucenic MJ, Carroll CL, et al: Pruritus in adult dermatomyositis. Clin Exp Dermatol 2004;29:273-276.

14 Hundley JL, Carroll CL, Lang W, et al: Cutaneous symptoms of dermatomyositis significantly impact patients' quality of life. J Am Acad Dermatol 2006;54:217-220.

15 Grover RW: Transient acantholytic dermatosis. Arch Dermatol 1970;101:426-434.

16 Guana AL, Cohen PR: Transient acantholytic dermatosis in oncology patients. J Clin Oncol 1994;12:1703-1709.

17 Kurzrock R, Cohen PR: Cutaneous paraneoplastic syndromes in solid tumors. Am J Med 1995;99:662-671.

18 Holdiness MR: Pruritus and the Leser-Trélat sign. J Am Acad Dermatol 1988;18:149.

19 Rampen HJ, Schwengle LE: The sign of LeserTrélat: does it exist? J Am Acad Dermatol 1989;21:50-55.

20 Zylicz Z, Krajnik M, Sorge AA, Costantini M: Paroxetine in the treatment of severe nondermatological pruritus: a randomized, controlled trial. J Pain Symptom Manage 2003;26: 1105-1112.

21 Davis MP, Frandsen JL, Walsh D, et al: Mirtazapine for pruritus. J Pain Symptom Manage 2003;25:288-291.

22 Hundley JL, Yosipovitch G: Mirtazapine for reducing nocturnal itch in patients with chronic pruritus: a pilot study. J Am Acad Dermatol 2004;50:889-891.

23 Puzantian T: Mirtazapine, an antidepressant. Am J Health Syst Pharm 1998;55:44-49.
24 Davis MP, Dickerson ED, Pappagallo M, et al: heir apparent to amitriptyline? Am J Hosp Palliat Care 2001;18:42-46.

25 Rose MA, Kam PC: Gabapentin: pharmacology and its use in pain management. Anaesthesia 2002;57:451-462.

26 Demierre MF, Taverna J: Mirtazapine and gabapentin for reducing pruritus in cutaneous T-cell lymphoma. J Am Acad Dermatol 2006; 55:543-544.

27 Magalhaes E, Mascarenhas AM, Kraychete DC, Sakata RK: Gabapentin to treat sacral perineural cyst-induced pain. Case report. Rev Bras Anestesiol 2004;54:73-77.

28 Dahl JB, Mathiesen O, Moiniche S: 'Protective premedication': an option with gabapentin and related drugs? A review of gabapentin and pregabalin in in the treatment of post-operative pain. Acta Anaesthesiol Scand 2004;48: 1130-1136.

29 Singhal S, Mehta J: Thalidomide in cancer. Biomed Pharmacother 2002;56:4-12.

30 Goncalves F: Thalidomide for the control of severe paraneoplastic pruritus associated with Hodgkin's disease. Am J Hosp Palliat Care 2010;27:486-487.

31 Singhal S, Mehta J, Desikan R, et al: Antitumor activity of thalidomide in refractory multiple myeloma. N Engl J Med 1999;341:1565-1571.

32 Bastuji-Garin S, Ochonisky S, Bouche P, et al: Incidence and risk factors for thalidomide neuropathy: a prospective study of 135 dermatologic patients. J Invest Dermatol 2002;119: 1020-1026.

33 Cowan A, Kehner G, Inan S: Targeting itch with ligands selective for $\kappa$ opioid receptors. Handb Exp Pharmacol 2015;226:291-314.

34 Ko MC: Neuraxial opioid-induced itch and its pharmacological antagonism. Handb Exp Pharmacol 2015;226:315-335. 
-35 Dawn AG, Yosipovitch G: Butorphanol for treatment of intractable pruritus. J Am Acad Dermatol 2006;54:527-531.

\$6 Zeng Z, Lu J, Shu C, et al: A comparision of nalbuphine with morphine for analgesic effects and safety: meta-analysis of randomized controlled trials. Sci Rep 2015;5:10927.

-37 Cohen SE, Ratner EF, Kreitzman TR, et al: Nalbuphine is better than naloxone for treatment of side effects after epidural morphine. Anesth Analg 1992;75:747-752.

38 Hawi A, Alcorn H Jr, Berg J, et al: Pharmacokinetics of nalbuphine hydrochloride extended release tablets in hemodialysis patients with exploratory effect on pruritus. BMC Nephrol 2015;16:47.
9 Andoh T, Nagasawa T, Satoh M, Kuraishi Y: Substance $\mathrm{P}$ induction of itch-associated response mediated by cutaneous NK1 tachykinin receptors in mice. J Pharmacol Exp Ther 1998; 286:1140-1145.

40 Hon KL, Lam MC, Wong KY, et al: Pathophysiology of nocturnal scratching in childhood atopic dermatitis: the role of brain-derived neurotrophic factor and substance P. Br J Dermatol 2007;157:922-925

-41 Duval A, Dubertret L: Aprepitant as an antipruritic agent? N Engl J Med 2009;361: 1415-1416.
42 Singer EM, Shin DB, Nattkemper LA, et al: IL-31 is produced by the malignant T-cell population in cutaneous T-cell lymphoma and correlates with CTCL pruritus. J Invest Dermatol 2013;133:2783-2785.

43 Cedeno-Laurent F, Singer EM, Wysocka M, et al: Improved pruritus correlates with lower levels of IL-31 in CTCL patients under different therapeutic modalities. Clin Immunol 2015;158:1-7.

44 Bates SE, Eisch R, Ling A, et al: Romidepsin in peripheral and cutaneous T-cell lymphoma: mechanistic implications from clinical and correlative data. Br J Haematol 2015;170:96-109. 